\title{
Benchmark test of drift-kinetic and gyrokinetic codes through neoclassical transport simulations
}

\author{
S. Satake ${ }^{\text {a }}$, Y. Idomura ${ }^{\text {b }}$ H. Sugama ${ }^{\text {a,c }}$, T.-H. Watanabe ${ }^{\mathrm{a}, \mathrm{c}}$ \\ ${ }^{a}$ National Institute for Fusion Science, 322-6, Orochi-cho, Toki, Gihu 509-5292, Japan \\ ${ }^{b}$ Japan Atomic Energy Agency, Higashi-Ueno 6-9-3, Taitou, Tokyo 110-0015, Japan \\ ${ }^{c}$ The Graduate University for Advanced Studies, 322-6, Orochi-cho, Toki, Gihu 509-5292, Japan
}

\begin{abstract}
Two simulation codes that solve the drift-kinetic or gyrokinetic equation in toroidal plasmas are benchmarked by comparing the simulation results of neoclassical transport. The two codes are the drift-kinetic $\delta f$ Monte Carlo code (FORTEC-3D) and the gyrokinetic full- $f$ Vlasov code (GT5D), both of which solve radially-global, five-dimensional kinetic equation with including the linear Fokker-Planck collision operator. In a tokamak configuration, neoclassical radial heat flux and the force balance relation, which relates the parallel mean flow with radial electric field and temperature gradient, are compared between these two codes, and their results are also compared with the local neoclassical transport theory. It is found that the simulation results of the two codes coincide very well in a wide rage of plasma collisionality parameter $v^{*}=0.01 \sim 10$ and also agree with the theoretical estimations. The time evolution of radial electric field and particle flux, and the radial profile of the geodesic acoustic mode frequency also coincide very well. These facts guarantee the capability of GT5D to simulate plasma turbulence transport with including proper neoclassical effects of collisional diffusion and equilibrium radial electric field.
\end{abstract}

Key words: Gyrokinetic, Drift-kinetic, Neoclassical transport

PACS: 52.65.Tt, 52.65.Ff, 52.25.Dg

\section{Introduction}

Kinetic approach to simulate transport phenomena in toroidal plasmas is the essential way in recent research. Two well-known models are the basis of these transport simulations. The one is the drift-kinetic model which mainly treats the diffusive process of particle and heat transport by Coulomb collisions among charged particles, or so-called neoclassical transport $[1,2,3]$, and the other is the gyrokinetic model $[4,5]$ which is mainly applied to study plasma turbulent transport caused by short wave-length electrostatic perturbations. Usually in experimental observations, the radial (direction across the minor radius of a toroidally confined plasma) heat transport level is one order higher compared to the prediction from neoclassical transport theory, which gives the irreducible minimal level of transport in a toroidally confined plasma. An explanation for this deterioration of plasma confinement is the micro-scale turbulence which is generated essentially in plasmas by thermodynamic forces like temperature gradient. The turbulence is also regulated by itself though a self-organized formation of a sheared $\mathbf{E} \times \mathbf{B}$ flow, or zonal flow in a plasma, and such non-linear microscopic turbulence in plasmas is described by the gyrokinetic model.

Although these two kinetic models have usually been treated separately in plasma transport analysis, the basic equations of these two models are derived from the same kinetic equation: the Boltzmann equation for a plasma distribution function $f$ in the six-dimensional phase space $(\mathbf{x}, \mathbf{v})$,

$$
\frac{\partial f}{\partial t}+\dot{\mathbf{x}} \cdot \frac{\partial f}{\partial \mathbf{x}}+\dot{\mathbf{v}} \cdot \frac{\partial f}{\partial \mathbf{v}}=C(f)
$$


where $C(f)$ denotes the Fokker-Planck collision operator. Both drift-kinetic and gyrokinetic models reduce one dimension from Eq. (1) by averaging over rapid gyration motion of charged particle around the magnetic field. Then particle motions in electromagnetic field is represented by the guiding-center motion. The main difference of these two models is that, in the gyrokinetic equation, the short scale perturbation of electrostatic potential, of which wave length is as short as $O\left(\rho_{i, e}\right)$ where $\rho_{i}\left(\rho_{e}\right)$ is the ion (electron) Larmor radius, is taken into account in the guiding-center equation of motion and in solving the Poisson equation, while such a short wave-length perturbation is neglected in a drift-kinetic model. On the other hand, macro-scale and slowly time-dependent physics such as toroidal rotation, fluxsurface averaged radial electric field, and the bootstrap current, of which scale lengths are comparable to the plasma temperature and density gradient scale lengths $L_{T}, L_{n}=|\nabla \ln T|^{-1},|\nabla \ln n|^{-1}$, are well described by the neoclassical transport theory. The equilibrium radial electric field $E_{r}$, which is determined self-consistently by the neoclassical process so that the plasma satisfies the quasi-neutrality, or the ambipolar condition, is also an important element to explain the plasma confinement especially in non-axisymmetric toroidal configurations[6, 7]. The strong $\mathbf{E} \times \mathbf{B}$ rotation reduces the radial ion flux, which is enhanced by the broken symmetry, to maintain plasma neutrality.

Recent progress in theory and simulation studies of plasma transport processes now turn their attention to the combination of these two models that can treat multi-scale transport phenomena ranging from $O\left(\rho_{i}\right)$ to $O\left(L_{T, n}\right)$. Idomura et al. [8] has developed a gyrokinetic simulation code GT5D which includes neoclassical physics by adopting the full- $f$ approach and introducing a conserved form linear Fokker-Planck collision operator[9, 10]. In the simulation study of ion-temperature-gradient (ITG) turbulence transport by GT5D, it is found that the shear of the equilibrium radial electric field $d E_{r} / d r$ plays a critical role in dictating the directions of avalanche propagation and non-diffusive momentum transport. Therefore, it is extremely important to determine experimentally relevant equilibrium radial electric fields $E_{r}$ also in gyrokinetic simulations. It is shown from the neoclassical theory that $E_{r}$ and the parallel mean flow satisfy a balance relation (see Eq.(20) in Sec.3). In this paper, we will focus on benchmarking this relation and the neoclassical heat diffusivity. It is noted that within the framework of the neoclassical theory, the parallel flows or the toroidal rotation is not determined in the lowest order theory because the particle flux is intrinsic ambipolar (In contrast, in helical systems, $E_{r}$ is dictated by the lowest order particle flux, which is not intrinsically ambipolar). In Ref.[11], it was shown that to estimate the neoclassical momentum transport, one need to calculate the momentum conservation with the toroidal viscosity up to higher order. However, in reality, one may need to determine parallel flows by the balance between external torque and turbulent transport, which are normally larger than the neoclassical momentum transport. These issues related to the momentum transport is out of scope in the present benchmark. It is important to check that the radial electric field is determined in the simulations to satisfy the force balance relation, no matter what parallel flow profile may appear.

In Ref.[8], a benchmark test of GT5D for the neoclassical transport calculation has been carried out, in which neoclassical heat diffusivity and the parallel force balance equation were tested against analytic formulae derived by neoclassical transport theories[1, 12]. Though it has shown qualitatively good agreements, more strict, quantitative test requires a strict drift-kinetic calculation as a counterpart, since analytic formulae use many conventional assumptions such as large-aspect-ratio expansion (assumption $a / R \ll 1$, where $a$ and $R$ are plasma minor and major radius, respectively) and zero-orbit-width limit (neglect of guiding-center radial excursion). Keeping the finiteness of the aspect ratio and orbit width of the radial guiding-center drift, which are called the finite-aspect-ratio and finiteorbit-width (FOW) effects, respectively, are related to the higher-order corrections of neoclassical transport which is essentially included in the gyrokinetic equation that GT5D solves, and these effects will be important in reactor-scale tokamak plasmas where the core temperature is high and the aspect-ratio is not so large. Therefore, to prove the applicability of GT5D to turbulence transport simulations with including neoclassical effects in a general situation, it is required to show that GT5D neoclassical transport simulation agrees with a drift-kinetic simulation which treats these higher-order effects correctly.

In the present paper, we report a new benchmark results of GT5D with a $\delta f$ neoclassical transport simulation code FORTEC-3D[13, 14], which has been developed to solve the drift-kinetic equation including the finite-aspectratio and FOW effects both in axisymmetric tokamak and helical plasmas. Neoclassical heat transport and parallel momentum balance are checked in the same way as shown in Ref. [8]. Not only the comparison of steady state transport feature, transient phase of radial electric field towards steady state is also compared between two codes. It will be shown that the frequency and Landau damping rate of geodesic acoustic mode (GAM), and the equilibrium $E_{r}$ also coincides each other.

Brief explanation on GT5D and FORTEC-3D codes are shown in Section 2. The case used for benchmark of 
neoclassical transport calculation is described in Section 3, where the analytic formulae to be used for the comparisons simulations with conventional neoclassical theory is also explained. The results and comparisons among two codes and neoclassical theory are presented in Section 4. Conclusions are given in Section 5.

\section{Calculation models}

\subsection{GT5D code}

GT5D code is a five-dimensional full- $f$ Vlasov code which solves a gyrokinetic equation with collision and source/sink terms,

$$
\frac{\partial \mathcal{J} f}{\partial t}+\nabla \cdot(\mathcal{J} \dot{\mathbf{R}} f)+\frac{\partial}{\partial v_{\|}}\left(\mathcal{J} \dot{v}_{\|} f\right)=\mathcal{J}\left(C(f)+S_{s r c}+S_{s n k}\right)
$$

where $f\left(\mathbf{R}, v_{\|}, \mu, t\right)$ is the guiding-center distribution function of ions, $\mathbf{R}$ is a position of the guiding center, $v_{\|}$is the parallel velocity, $\mu$ is the magnetic moment, and $\mathcal{J}$ is the Jacobian of the guiding-center coordinates. The nonlinear characteristics $\left(\dot{\mathbf{R}}, \dot{v}_{\|}\right)$are given by the gyro-center Hamiltonian as follows:

$$
\begin{aligned}
H & =\frac{1}{2} m v_{\|}^{2}+\mu B+e\langle\phi\rangle_{\alpha}, \\
\dot{\mathbf{R}} \equiv\{\mathbf{R}, H\} & =v_{\|} \mathbf{b}+\frac{c}{e B * \|} \mathbf{b} \times\left(e\langle\phi\rangle_{\alpha}+m v_{\|}^{2} \mathbf{b} \cdot \nabla \mathbf{b}+\mu \nabla B\right), \\
v_{\|} \equiv\left\{v_{\|}, H\right\} & =-\frac{\mathbf{B}^{*}}{m B * \|} \cdot\left(e\langle\phi\rangle_{\alpha}+\mu \nabla B\right),
\end{aligned}
$$

where $\mathbf{B}=B \mathbf{b}$ is the magnetic field, $\mathbf{B}^{*}=\mathbf{B}+\left(B v_{\|} / \Omega\right) \nabla \times \mathbf{b}, B *_{\|}=\mathbf{b} \cdot \mathbf{B}^{*}, \Omega=e B /(m c)$ is the cyclotron frequency, and the gyro-averaging operator is defined as $\langle\cdot\rangle_{\alpha}=\oint \cdot d \alpha / 2 \pi$ where $\alpha$ is the gyro-phase angle. The gyrokinetic Poisson bracket operator $\{F, G\}$ is defined as follows:

$$
\begin{aligned}
\{F, G\} \equiv & \frac{\Omega}{B}\left(\frac{\partial F}{\partial \alpha} \frac{\partial G}{\partial \mu}-\frac{\partial G}{\partial \alpha} \frac{\partial F}{\partial \mu}\right) \\
& +\frac{\mathbf{B}^{*}}{m B * \|} \cdot\left(\nabla F \frac{\partial G}{\partial v_{\|}}-\nabla G \frac{\partial F}{\partial v_{\|}}\right) \\
& -\frac{c}{e B * \|} \mathbf{b} \cdot \nabla F \times \nabla G .
\end{aligned}
$$

The self-consistency is imposed by the quasi-neutrality condition or the gyrokinetic Poission equation

$$
\begin{array}{r}
-\nabla_{\perp} \cdot \frac{\rho_{t i}^{2}}{\lambda_{D i}^{2}} \nabla_{\perp} \phi+\frac{1}{\lambda_{D e}^{2}}\left(\phi-\langle\phi\rangle_{f}\right) \\
=4 \pi e\left[\int f \delta([\mathbf{R}+\rho]-\mathbf{x}) d^{6} Z-n_{0 e}\right],
\end{array}
$$

where $\mathbf{R}+\rho$ is a particle position, $d^{6} Z=\mathcal{J} d \mathbf{R} d v_{\|} d \mu d \alpha=m^{2} B *_{\|} d \mathbf{R} d v_{\|} d \mu d \alpha$ is the phase space volume, $\rho_{t i}$ is the Larmor radius evaluated with the thermal velocity $v_{t i}, \lambda_{D i}, \lambda_{D e}$ are ion and electron Debye lengths, and $\langle\cdot\rangle_{f}$ is a flux surface averaging operator, respectively.

The Fokker-Planck collision operator is linearized and separated into the test-particle and field-particle parts as $C(f)=C_{T}(f)+\mathcal{P} f_{M}$, where $C_{T}$ is given by a second-order differentiation operator in the velocity space[9],

$$
\begin{aligned}
C_{T}(f)= & \frac{\partial}{\partial s}\left(v_{\perp s} v^{2} f\right)+\frac{\partial}{\partial u}\left(v_{s \|} u f\right)+\frac{1}{2} \frac{\partial^{2}}{\partial s^{2}}\left(v_{\perp} v^{4} f\right) \\
& +\frac{1}{2} \frac{\partial^{2}}{\partial u^{2}}\left(v_{\|} v^{2} f\right)+\frac{\partial^{2}}{\partial s \partial u}\left(v_{\| \perp} v^{3} f\right)
\end{aligned}
$$


where $s=2 \mu B / m$ and $u=v_{\|}-U_{\|}$are a moving frame with respect to the mean parallel flow $U_{\|}$, and $v^{2}=u^{2}+s$. The field particle operator $\mathcal{P} f_{M}$ is explained in Section 2.2. Note that the collision operator used in GT5D is a drift-kinetic limit, in which neither the finite-Larmor-radius (FLR) effect nor the gyrophase angle dependency is considered.

In GT5D, the gyrokinetic Poisson bracket operator is discretized using the fourth order NDCFD[15], and the gyrokinetic Poisson equation (5) is computed using toroidal mode expansion and a 2D finite element approximation on the poloidal plane. The linearized collision operator is discretized using the sixth order centered finite difference. The time integration is performed using the second order additive semi-implicit Runge-Kutta method[16] and a stiff linear term involving the parallel streaming is treated implicitly. An implicit part is solved using the generalized conjugate residual method, and a typical time step width is given as $\Delta t=10 \Omega^{-1}$.

\subsection{FORTEC-3D code}

FORTEC-3D is a five-dimensional $\delta f$ Monte Carlo code which solves the drift-kinetic equation for perturbed distribution function $\delta f=f-f_{M}$,

$$
\begin{aligned}
\frac{D}{D t} \delta f & \equiv \frac{\partial}{\partial t} \delta f+\left(\mathbf{v}_{\|}+\mathbf{v}_{d}\right) \cdot \nabla \delta f+\dot{\mathcal{K}} \frac{\partial \delta f}{\partial \mathcal{K}}-C_{T}(\delta f) \\
& =-\left(\mathbf{v}_{d} \cdot \nabla+\dot{\mathcal{K}} \frac{\partial}{\partial \mathcal{K}}\right) f_{M}+\mathcal{P} f_{M} .
\end{aligned}
$$

where $f_{M}$ is a time-independent local Maxwellian which has constant $n$ and $T$ on a flux surface, $\mathbf{v}_{d} \equiv \dot{\mathbf{R}}-v_{\|} \mathbf{b}$ is the drift velocity, and the independent velocity variables are chosen here as $\left(\mathcal{K}=m v^{2} / 2, \mu\right)$. The linearized Fokker-Planck collision operator is the same one as used in GT5D. To be applicable to general non-axisymmetric magnetic field configurations, FORTEC-3D adopts the Boozer magnetic coordinates[17] and a Hamiltonian formalism of the guiding-center equation in the Boozer coordinates.[18, 19] The main difference between the drift-kinetic and gyrokinetic equations is that the electrostatic potential in drift-kinetic model is the one evaluated on the guidingcenter position, not a gyro-averaged one. And for neoclassical transport calculation, only the radial electric field $E_{r}=-d\langle\phi\rangle_{f} / d r$ is considered, where $r$ denotes the radial coordinate and $\langle\phi\rangle_{f}$ is the averaged electrostatic potential of a flux-surface $r=$ const. Therefore, the drift-kinetic model can be considered as an approximation of gyrokinetic model by replacing $\langle\phi\rangle_{\alpha} \rightarrow\langle\phi\rangle_{f}$. Without any external source considered in FORTEC-3D, the time change of the kinetic energy is given by $\dot{\mathcal{K}}=e \mathbf{v}_{d} \cdot \mathbf{E}_{r}$. The terms $\mathbf{v}_{d} \cdot \nabla \delta f$ and $\dot{\mathcal{K}} \partial \delta f / \partial \mathcal{K}$ represent the FOW effects, which are neglected in the local transport theory.

FORTEC-3D adopts a two-weight scheme of $\delta f \operatorname{method}[20,21]$. The perturbed distribution function is expressed by simulation markers with its weight $w ; \delta f=w \cdot g$, where $g=\sum_{k} \delta\left(\mathbf{R}_{k}-\mathbf{R}\right) \delta\left(\mathcal{K}_{k}-\mathcal{K}\right) \delta\left(\mu_{k}-\mu\right) / \mathcal{J}_{\mathcal{K}}$ is the distribution function of simulation markers and $\mathcal{J}_{\mathcal{K}}=2 \pi \mathcal{J} /\left(m\left|v_{\|}\right|\right)$, while another weight $p$ is defined so that it satisfies the relation $f_{M}=p \cdot g$. The test-particle collision operator is expressed by random kicks of marker velocity in the $\left(v_{\|}, v_{\perp}\right)$-space[9] so that it reproduce the diffusion process described by Eq. (6). Since each simulation marker moves according to the 1.h.s. of Eq. (7), or $D g / D t=0$, time evolution of marker weights are given by

$$
\begin{aligned}
\dot{w} & =-\frac{p}{f_{M}}\left[\mathbf{v}_{d} \cdot \nabla+\dot{\mathcal{K}} \frac{\partial}{\partial \mathcal{K}}-\mathcal{P}\right] f_{M}, \\
\dot{p} & =\frac{p}{f_{M}}\left[\mathbf{v}_{d} \cdot \nabla+\dot{\mathcal{K}} \frac{\partial}{\partial \mathcal{K}}\right] f_{M},
\end{aligned}
$$

where the auxiliary weight $p$ is used to replace $1 / g$ with $p / f_{M}$. In the collisionless limit, we have $\dot{p}=-\dot{w}$ and simple one-weight $\delta f$ method is reproduced. The two-weight scheme is required in a system with collisional dissipation where the evolution of the marker distribution function cannot be described only by the Hamiltonian, e. g., $D g / D t=0$ but $\partial g / \partial t+\{g, H\} \neq 0$. It is also noted that since the background $f_{M}$ is fixed in time, the $\delta f$ scheme can be applied for a shorter time scale than the plasma profiles of background $n$ and $T$ changes substantially. For a magnetic confined plasma, this time scale is usually much longer than the typical collision time at which neoclassical quasi-steady state is achieved, and then the $\delta f$ approach is held valid unless there is extremely steep gradient in the background plasma profile. 
The linearized Fokker-Plank collision operator in GT5D and FORTEC-3D is made to satisfy the following relations

$$
\begin{array}{r}
\int d^{3} v \mathcal{M}\left(C_{T}(f)+\mathcal{P} f_{M}\right)=0 \text { for } \mathcal{M}=\left\{1, v_{\|}, v^{2}\right\}, \\
C_{T}(f)+\mathcal{P} f_{M}=0 \text { for } \delta f=\left(c_{0}+c_{1} v_{\|}+c_{2} v^{2}\right) f_{M}, c_{i} \in R,
\end{array}
$$

which describe the conservation property and the H-theorem for linearized collision operator, respectively. Note that $C_{T}(f)$ in Eq. (9) is replaced by $C_{T}(\delta f)$ in the $\delta f$-method, and as it is mentioned in Sec. 2.1, no FLR-effect is considered and an identification $\mathcal{J} d v_{\|} d \mu d \alpha \simeq d^{3} v$ is used in Eq. (9a). Though numerical implementation of the test-particle operator is different between GT5D Vlasov code and FORTEC-3D Monte Carlo code, the field particle operator is given in the same form as follows[10, 20]:

$$
\begin{aligned}
\mathcal{P} & =-\frac{1}{n}[a F(x)+b G(x) \xi+c H(x)], \\
F(x) & =1-3 \sqrt{\frac{\pi}{2}} x^{-1 / 2}\left(\phi(x)-\phi(x)^{\prime}\right), \\
G(x) & =3 \sqrt{\frac{\pi}{2}} x^{-1 / 2} \phi(x), \\
H(x) & =3 \sqrt{\frac{\pi}{2}} x^{-1 / 2}\left(\phi(x)-\phi(x)^{\prime}\right),
\end{aligned}
$$

where $x=v^{2} / v_{t h}^{2}, \xi=v_{\|} / v$, and $\phi(x)=(2 / \sqrt{\pi}) \int_{0}^{x} d t e^{-x^{\prime}} \sqrt{x^{\prime}}$ is the Maxwellian integral, respectively. The factors $(a, b, c)$ are determined by the change of particle number, momentum, and energy caused by the test-particle operator, e. g.,

$$
\begin{aligned}
\{a, b, c\} & =\left\{\delta n, 2 \delta P / v_{t h}, 2 \delta E /\left(3 v_{t h}^{2}\right)\right\}, \\
\{\delta n, \delta P, \delta E\} & =\int d^{3} v\left\{1, v_{\|}, v^{2}\right\} C_{T}(f) .
\end{aligned}
$$

In practice, however, this field-particle operator does not exactly satisfy the conservation property Eq. (9a) either in Vlasov code with a finite velocity grids or in Monte Carlo code with a finite number of simulation markers. Then an iterative operation of the field-particle operator is usually taken in simulation codes[8, 20] to reduce the numerical error, but it is time consuming. In FORTEC-3D, the accuracy of collision operator is improved by solving the factors $(a, b, c)$ as functionals of $(\delta n, \delta P, \delta E)$ instead of using their theoretical values given by Eq. (11) so that the linearized collision operator exactly satisfies the conservation law[14]. By substituting Eqs. (10) and (12) into Eq. (9a) and using the relation $f_{M}=p \cdot g$, we have

$$
\left(\begin{array}{c}
a \\
b \\
c
\end{array}\right)=-\sum_{k}\left(\begin{array}{ccc}
F_{k} p_{k} & G_{k} \xi_{k} p_{k} & H_{k} p_{k} \\
F_{k} x_{k}^{1 / 2} \xi_{k} p_{k} & G_{k} x_{k}^{1 / 2} \xi_{k}^{2} p_{k} & H_{k} x_{k}^{1 / 2} \xi_{k} p_{k} \\
F_{k} x_{k} p_{k} & G_{k} x_{k} \xi_{k} p_{k} & H_{k} x_{k} p_{k}
\end{array}\right)^{-1} \cdot\left(\begin{array}{c}
\delta n \\
\delta P / v_{t h} \\
\delta E / v_{\text {th }}^{2}
\end{array}\right)
$$

where the summation is taken over markers (index $k$ ) within a small volume cell, and abbreviations $F_{k}=F\left(x_{k}\right)$ and so on are used. Note that $\delta n$ etc. are also evaluated by integrating Eq. (12) over the same small cell. With this improved field-particle operator, the numerical error in collision operator is suppressed to the rounding-error level in FORTEC-3D[14]. Recently GT5D has also adopted the same idea for the field-particle operator (in GT5D, Eq. (13) is computed at each spatial grid with the summation over markers replaced by an integral over the velocity space) and succeeded in reducing calculation time compared to the previous iterative operation.

The time evolution of the radial electric field is solved according to the radial current as follows,

$$
\begin{gathered}
-\left\langle\frac{\rho_{t i}^{2}}{4 \pi e \lambda_{D i}^{2}}\right\rangle_{f} \frac{\partial E_{r}}{\partial t}=\Gamma_{i}-\Gamma_{e}, \\
\Gamma_{i}=\left\langle\int \mathcal{J}_{\mathcal{K}} d \mathcal{K} d \mu \mathbf{v}_{d} \cdot \nabla r \delta f_{i}\right\rangle_{f} . \\
5
\end{gathered}
$$


Note that the local Maxwellian $f_{M}$ does not contribute radial flux. Since it is time consuming to solve electron dynamics and the FOW effect is negligible for electron transport, FORTEC-3D only solves the ion drift-kinetic equation (7) using the $\delta f$ method. For electrons, a reduced local neoclassical transport model for helical magnetic field configurations is adopted $[13,22]$. In axisymmetric tokamak case here, we simply neglect the electron radial flux $\Gamma_{e}$ which is $O\left(\sqrt{m_{e} / m_{i}}\right)$ smaller than $\Gamma_{i}$ in any case. Similarly, since the ion-electron collision is also $O\left(\sqrt{m_{e} / m_{i}}\right)$ smaller than the ion-ion self collision, the ion-electron collision term is neglected in Eq. (7).

In FORTEC-3D, time integral is performed in two different time steps, one is for marker guiding-center motion and radial electric field, and the other is for collision operator. Typical time steps are given as $\Delta t_{o} \sim q R_{0} /\left(100 v_{t i}\right)$ for the former and $\Delta t_{c} \sim \tau_{i i} / 1000$ for the latter, respectively, where $q$ is a safety factor of the magnetic field, $R_{0}$ is the major radius of the magnetic axis, and $\tau_{i i}$ is a typical 90-degree scattering time of ion-ion collisions. The marker orbital motion is solved by fourth-order Runge-Kutta method and the radial electric field is integrated by a first-order modified Euler method. Time integral of $C_{T}$ and $\mathcal{P} f_{M}$ are performed by first order Euler method. To reduce statistical noise unavoidable in any Monte Carlo methods, an averaging scheme of marker weights[21] in the phase space, a filtering scheme for nonphysically large weights[14, 23], and a marker recycling scheme for those markers filtered out are introduced, which are deliberately operated not to affect the conservation properties or time-averaged observable values such as $\Gamma_{i}$ and $U_{\|}$.

\subsection{Drift-kinetic limit of gyrokinetic system}

In considering a way of benchmarking between gyrokinetic and drift-kinetic simulations, it is to be noted that the gyrokinetic Vlasov-Poisson system, equations (2) and (5) reduces to the drift-kinetic system equations (7) and (14), in the limit only the macroscopic radial electric field $E_{r}=-d\langle\phi\rangle_{f} / d r$ is considered. Firstly, for the macroscopic perturbation $k_{\perp} L_{n, T} \sim O(1)$, the FLR effect is negligible and the gyrokinetic equation (2) reduces to the drift-kinetic equation (7). The apparent difference between Eqs. (2) and (7) can be resolved by transforming the drift-kinetic equation in the conserved form using the incompressible condition of the Hamiltonian flow, $\partial \mathcal{J}_{\mathcal{K}} / \partial t+\nabla \cdot\left(\mathcal{J}_{\mathcal{K}} \dot{\mathbf{R}}\right)+\partial\left(\mathcal{J}_{\mathcal{K}} \dot{\mathcal{K}}\right) / \partial \mathcal{K}=0$, which holds true both in the drift-kinetic and gyrokinetic equations with the Hamiltonian formulation of the guidingcenter motion[18]. Secondly, by taking a time derivative and flux-surface averaging of Eq. (5) and substituting Eq. (2), we have

$$
\frac{\partial}{\partial r}\left[-\left\langle\frac{\rho_{t i}^{2}}{4 \pi e \lambda_{D i}^{2}}\right\rangle_{f} \frac{\partial E_{r}}{\partial t}-\Gamma_{i}\right]=\left\langle\int \mathcal{J} d v_{\|} d \mu d \alpha C(f)\right\rangle_{f}=0,
$$

where the source and sink terms, and electron radial flux are neglected for simplicity, and the conservation property of collision term is used in the last equality. The ion radial flux is given by

$$
\begin{aligned}
\Gamma_{i} & \equiv\left\langle\int d^{6} Z f_{i}\left(\dot{\mathbf{R}}_{0} \cdot \nabla r\right) \delta([\mathbf{R}+\rho]-\mathbf{x})\right\rangle_{f} \\
& \simeq\left\langle 2 \pi \int d v_{\|} d \mu \mathcal{J} f_{i}\left(\dot{\mathbf{R}}_{0} \cdot \nabla r\right)\right\rangle_{f} \\
& =\left\langle\int d \mathcal{K} d \mu \mathcal{J}_{\mathcal{K}} \delta f_{i}\left(\mathbf{v}_{d} \cdot \nabla r\right)\right\rangle_{f} .
\end{aligned}
$$

In this equation, we use the approximation $\mathbf{R}+\rho \simeq \mathbf{R}$ and $\dot{\mathbf{R}}_{0}$ is the guiding-center orbit given by Eq. (3) with an approximation $\langle\phi\rangle_{\alpha} \simeq\langle\phi\rangle_{f}$, and one can see that the last form is identical to Eq. (14b). Thus gyrokinetic and driftkinetic is identical in the macroscopic perturbation limit. Therefore, to compare the neoclassical transport calculation with FORTEC-3D, all the benchmarks of GT5D shown in Section 4 are conducted in plasmas where the FLR effect is negligibly small, i.e., $\rho_{i} / L \ll 1$, and only the flux-surface averaged potential $\langle\phi\rangle_{f}$ is solved.

It is also to be noted that the term $\left(\phi-\langle\phi\rangle_{f}\right) / \lambda_{D e}^{2}$ appeared in the 1.h.s. of Eq. (5) vanishes in Eq. (15) in the approximation $\phi \rightarrow\langle\phi\rangle_{f}$. This means that the adiabatic response of electron along magnetic field lines is ignored in the drift-kinetic limit. Then the drift-kinetic model can also be regarded as a $T_{e} / T_{i} \rightarrow 0$ and $\rho_{i} / L \ll 1$ limit of the gyrokinetic model. This property is used in later in Section 3 to derive analytic estimation for GAM frequency and damping rate in a drift-kinetic system. 


\section{Case for benchmark}

For the benchmark of neoclassical transport calculation, we set two types of circular cross-section concentric tokamak geometries. The one has a relatively fat aspect ratio, $\epsilon_{a}^{-1} \equiv R_{0} / a=1.31 \mathrm{~m} / 0.47 \mathrm{~m}=2.79$. It is called "the case 1" here and hereafter. Initial plasma distribution function is given by local Maxwellian, and the density, temperature, safety factor, and the magnetic field are given by the following form

$$
\begin{aligned}
n(x) & =n_{0}\left[-\frac{\Delta_{n}}{L_{n}} \tanh \left(\frac{x-0.5}{\Delta_{n} / a}\right)\right], \\
T_{i}(x) & =T_{i 0}\left[-\frac{\Delta_{T i}}{L_{T i}} \tanh \left(\frac{x-0.5}{\Delta_{T i} / a}\right)\right], \\
q(x) & =0.854+2.184 x^{2}, \\
\mathbf{B}(x, \theta) & =\frac{B_{0}}{1+x \epsilon_{a} \cos \theta}\left(\mathbf{e}_{\zeta}+\frac{x \epsilon_{a}}{q(x)} \mathbf{e}_{\theta}\right),
\end{aligned}
$$

where $x=r / a, \theta$ is the poloidal angle, and $\mathbf{e}_{\zeta}$ and $\mathbf{e}_{\theta}$ are toroidal end poloidal unit vector, respectively. In the case 1, we intend to check the radial dependence of neoclassical transport in a low-collisionality plasma, and the profile has relatively broader ion temperature gradient $\left(T_{i 0}=2.12 \mathrm{keV}, L_{T i}=R_{0} / 10.0, \Delta_{T i}=0.30 a, n_{0}=5.0 \times 10^{19} \mathrm{~m}^{-3}, L_{n}=\right.$ $R_{0} / 2.20, \Delta_{n}=0.30 a$ ) as shown in figure. 1 . The effective collisionality[1] is small in almost the entire plasma, $v_{*} \equiv \sqrt{2} q R_{0} /\left(\epsilon^{3 / 2} \tau_{i i} v_{t i}\right)<0.1$, where $v_{t i} \equiv \sqrt{2 T_{i} / m_{i}}$ and $\epsilon=r / R_{0}$. It corresponds to a banana regime plasma.

In the other case, we use a more slim configuration than the case $1, R_{0} / a=2.35 \mathrm{~m} / 0.47 \mathrm{~m}=5.0$, in order that comparisons of simulation results with the large-aspect-ratio limit neoclassical theory be more plausible. The gradient and collisionality are set equal on the $r=0.5 a$ surface both in GT5D and FORTEC-3D simulations $\left(L_{T i}=\right.$ $L_{n}=R_{0} / 6.0, \Delta_{T i}=\Delta_{n}=0.20 a, v_{*} \simeq 0.012$ ) and it is called "the case 2 ". To benchmark the collisionality dependence of neoclassical transport, the collisionality is artificially multiplied by 10,100 , and 1000 times from that of the case 2 , and these are called the case 3,4 , and 5 , respectively. In the all cases above, electron temperature profile is properly given but it is irrelevant to the benchmarks performed here, since no electron dynamics is involved. In each case, typical Larmor radius scale is small, $\rho_{t i} / a \simeq 1 / 150$, enough to apply the drift-kinetic model where the FLR effect is regarded as negligibly small. However, it is to be noted that the physics concerning the finite-orbit-width effect and finite-aspect-ratio effect are retained in both gyrokinetic and drift-kinetic calculations and that the FLR effect is retained in solving the gyrokinetic equation in GT5D though it is small in the present cases.

What are observed and compared with neoclassical theory in the benchmarks are as follows: Firstly, neoclassical radial ion heat diffusivity $\chi_{i}$ is compared. It is evaluated in GT5D and FORTEC-3D codes from radial heat flux,

$$
Q_{i}=\left\langle\int d v_{\|} d \mu d \alpha \mathcal{J} f_{i}\left(\dot{\mathbf{R}}_{0} \cdot \nabla r\right)\left(\frac{1}{2} m_{i} v_{\|}^{2}+\mu B\right)\right\rangle_{f}-\frac{5}{2} \Gamma_{i} T_{i},
$$

where $d v_{\|} d \mu d \alpha \mathcal{J} f_{i}$ is replaced by $d \mathcal{K} d \mu \mathcal{J}_{\mathcal{K}} \delta f_{i}$ in FORTEC-3D. With taking a time average of $Q_{i}$ after it settled in a quasi-steady state, $\chi_{i}$ is given by $\chi_{i}=-Q_{i} /\left(n_{i} \nabla T_{i}\right)$. Theoretic value of $\chi_{i}$ is given by the Chang-Hinton model as follows,

$$
\begin{aligned}
\chi_{i}= & \epsilon^{1 / 2} \frac{\rho_{i \theta}^{2}}{\tau_{i i}} K_{2}, \\
K_{2}= & \left(\frac{0.66+1.88 \epsilon^{1 / 2}-1.54 \epsilon}{1+1.03 v_{*}^{1 / 2}+0.31 v_{*}}\right)\left\langle\frac{B_{0}^{2}}{B^{2}}\right\rangle_{f} \\
& +\frac{0.79}{\epsilon^{1 / 2}}\left(\frac{0.74 v_{*} \epsilon^{3 / 2}}{1+0.74 v_{*} \epsilon^{3 / 2}}\right)\left[\left\langle\frac{B_{0}^{2}}{B^{2}}\right\rangle_{f}-\left\langle\frac{B^{2}}{B_{0}^{2}}\right\rangle_{f}^{-1}\right],
\end{aligned}
$$

where $\rho_{i \theta} \equiv q \rho_{t i} / \epsilon$ is the poloidal Larmor radius. This formula is constructed by using expansion in small parameter $\epsilon$ and by connecting analytic solutions both in the $v_{*} \ll 1$ and $v_{*} \gg 1$ limits[12]. It contains the leading-order correction of the finite-aspect-ratio effect and the Shafranov shift[24]. However, the latter effect is neglected in the benchmark because a concentric flux surface configuration, which corresponds to the zero plasma-beta limit, is used. 
Secondly, we check the force balance relation among the parallel flow, the Pfirsh-Schlüter flow, and the neoclassical poloidal flow. A force balance relation in the local neoclassical theory[1] is given by

$$
\left\langle U_{\| i} B\right\rangle_{f}=\frac{I T_{i}}{e}\left(\frac{d \psi}{d r}\right)^{-1}\left[(k-1) \frac{d}{d r} \ln T_{i}-\frac{d}{d r} \ln n_{i}+\frac{e E_{r}}{T_{i}}\right],
$$

where $\psi(r)$ is the poloidal flux and $I(r)=R B_{t}$. The factor $k$ is the same one as $\left(\beta_{1}, g_{2 i}\right)$ appeared in Eq. (6.134) in Ref. [1], which depends on the collisionality $v_{*}$. Approximate fit formula for a wide range of $v_{*}$ is given as follows:

$$
k \simeq\left(\frac{1.17-0.35 v_{*}^{1 / 2}}{1+0.7 v_{*}^{1 / 2}}-2.1 v_{*}^{2} \epsilon^{3}\right) /\left(1+v_{*}^{2} \epsilon^{3}\right) .
$$

This formula, however, is constructed in the $\epsilon \rightarrow 0$ limit for $v_{*} \ll 1$. To check the finite-aspect-ratio correction, we also use a more precise evaluation of $k$-factor, which can be obtained by directly evaluating $k\left(v_{*}, \epsilon\right)$ from neoclassical transport theory. According to the moment equation approach in Ref. [2], it is given as follows:

$$
k=\frac{-\sqrt{2} \hat{\mu}_{2}}{\sqrt{2} \hat{\mu}_{1}+\left(f_{t} / f_{c}\right)\left(\hat{\mu}_{1} \hat{\mu}_{3}-\hat{\mu}_{2}^{2}\right)}
$$

where $f_{t} \simeq 1.46 \sqrt{\epsilon}$ is the trapped particle fraction, $f_{c}=1-f_{t}$, and the viscosity coefficients $\hat{\mu}_{j}\left(v_{*}, \epsilon\right)$ for ions are given by

$$
\begin{aligned}
& \left\{\begin{array}{l}
\hat{\mu}_{1}=\hat{K}_{11}, \\
\hat{\mu}_{2}=\hat{K}_{12}-\frac{5}{2} \hat{K}_{11}, \\
\hat{\mu}_{3}=\hat{K}_{13}-5 \hat{K}_{12}+\frac{25}{4} \hat{K}_{11},
\end{array}\right. \\
& \hat{K}_{i j}=\left\{x^{2(i+j-2)} v_{t o t} \tau_{i i}\right\},
\end{aligned}
$$

where $x^{2}=m v^{2} /\left(2 T_{i}\right),\{A(x)\} \equiv(8 / 3 \sqrt{\pi}) \int_{0}^{\infty} d x \exp \left(-x^{2}\right) x^{4} A(x)$, and $v_{t o t}$ is given by Eq. (4.73) in Ref. [2]. For the benchmarks, $\hat{K}_{i j}$ are numerically evaluated according to the definition above rather than using the fitting formula of them as shown in Ref. [2] or [25]. We benchmark the force balance relation in the simulation by solving Eq. (20) for $k$ by the observed values of $\left\langle U_{\| i} B\right\rangle_{f}$ and $E_{r}$ with given $\nabla n$ and $\nabla T_{i}$ and comparing it with the theory.

Thirdly, to check the temporal behavior, the time evolution of the radial electric field and ion flux are compared between two codes. An important feature we can see commonly in both these two kinetic systems is the geodesic acoustic mode (GAM) oscillation and collisionless Landau damping of it. In a gyrokinetic analysis[26], the real frequency and the damping rate of GAM in a circular tokamak geometry are approximately given as follows,

$$
\begin{aligned}
\omega_{G}= & \frac{\sqrt{7}}{2}\left(\frac{v_{t i}}{R_{0}}\right) \sqrt{1+\frac{46}{49 q^{2}}}, \\
\gamma= & -\frac{q v_{t i} \sqrt{\pi}}{2 R_{0}}\left(1+\frac{46}{49 q^{2}}\right)^{-1}\left[\exp \left(-\hat{\omega}_{G}^{2}\right)\left\{\hat{\omega}_{G}^{4}+\hat{\omega}_{G}^{2}\right\}\right. \\
& \left.+\frac{1}{32}\left(q k_{r} \rho_{t i}\right)^{2} \exp \left(-\hat{\omega}_{G}^{2} / 4\right)\left\{\frac{\hat{\omega}_{G}^{6}}{16}+\frac{\hat{\omega}_{G}^{4}}{2}+3 \hat{\omega}_{G}^{2}\right\}\right],
\end{aligned}
$$

where $\hat{\omega}_{G} \equiv q R_{0} \omega_{G} / v_{t i}$, and the drift-kinetic limit $\tau_{e} \equiv T_{e} / T_{i} \rightarrow 0$ is taken from the original analytic formula. The benchmark cases, in which a drift-kinetic limit where the FLR effect and electron adiabatic response are neglected, correspond to the case $\tau_{e} \rightarrow 0$ limit is taken, as is explained Section 2.3. The first term on the r.h.s. of Eq. (26) is the Landau damping in the zero-orbit-width limit and the second term is due to the resonance of passing particles with the GAM at $\left|v_{\|}\right|=R q \omega / 2$ caused by the finiteness of orbit widths. We compare the real frequency and damping rate of GAM between the theory and those evaluated by taking power spectrum of $E_{r}$ oscillation observed in GT5D and FORTEC-3D simulations. 


\section{Benchmark results}

First, benchmark results for the case 1 is shown. After a time $t / \tau_{i i} \sim 1$, the neoclassical ion heat flux and the force balance reach an equilibrium state. The radial profile of the ion heat diffusivity $\chi_{i}$ and the neoclassical force balance factor $k$ in Eq. (20) are plotted in figures 2 and 3. The estimations of these values from conventional neoclassical theory explained in the previous section are also plotted in the figures. Comparing the evaluated $\chi_{i}$ and the $k$-factor, we find good coincidence between the two simulations almost the entire region of plasma minor radius. Concerning $\chi_{i}$, these two simulation results agree very well, though the discrepancy from the Chang-Hinton's formula is increasing toward the two regions $r / a \rightarrow 1$ and $r / a \rightarrow 0$. These differences are attributed to the finite-aspect-ratio and finiteorbit-width effects respectively, which are neglected or approximated in the analytic formula. In the outer region, the inverse aspect ratio $\epsilon$ becomes larger $(\epsilon \sim 0.3$ in the case 1), and the analytic formula, which uses a expansion in $\epsilon$, is not a good approximation there. On the other hand, in the region close to the magnetic axis, it is known that wide width guiding-center orbits called "potato orbits" appear. In Ref. [27], it is shown that the FOW effect of potato orbit affects the neoclassical heat diffusivity in the region $r \lesssim 2\left(q^{2} \rho_{i}^{2} R_{0}\right)^{1 / 3}$, which in the case 1 becomes $r \simeq 0.20 a$, and reduces $\chi_{i}$ inside the region if the temperature gradient near the magnetic axis is not steep. This potato-orbit effect can explain the smaller $\chi_{i}$ of kinetic simulations in the core region. Concerning the factor $k$, these two kinetic simulations agrees well with Eq. (22), which is direct evaluation of $k\left(v_{*}, \epsilon\right)$ from a finite-aspect-ratio theory, rather than the Hinton-Hazeltine's large-aspect-ratio limit (21). One can see the large discrepancy between simulation results and the estimation by Eq. (21), which suggests the importance of the finite-aspect-ratio effect on the force balance relation in a low-collisionality plasma.

Second, collisionality dependence of $\chi_{i}$ and $k$ at the $r=0.5 a$ surface are scanned for the cases 2 to 5 , which are shown in figures 4 and 5. Since the GAM damping rate is seldom affected by the collisionality, it takes longer to achieve a steady state value of transport coefficients as $v_{*}$ increases, if it is seen in a normalized time unit $\bar{t}=t / \tau_{i i}$. Then the timings we take the averaged values of $\chi_{i}$ and $k$ are varied according to $v_{*}$. Very good agreements between GT5D and FORTEC-3D results, as well as good coincidences with neoclassical theories, can be seen. In real plasma confinement experiments, the plasma collisionality $v_{*}$ sometimes varies largely toward the plasma edge, and it is important for a global simulation code as benchmarked here to have an adaptability to a wide range of $v_{*}$. Concerning the force balance relation, as it is seen in the case 1, it is found that the finite-aspect-ratio correction to the factor $k$ is effective for low collisionality cases, even with relatively small $\epsilon \simeq 0.1$ for the cases 2 to 5 .

Next, let us compare the temporal behavior of the simulations. Figures 6 and 7 show transient evolution of the ion particle flux $\Gamma_{i}$ and radial electric field $E_{r}$ observed in two codes for the case 3, at $r=0.5 a$. Analytic estimation of the GAM oscillation from Eqs. (25) and (26) is also plotted in Fig. 6, where $\Gamma_{i}(t)=\Gamma_{0} \sin \left(\omega_{G} t\right) \exp (\gamma t)$ is assumed. Here we omit the FOW effect in the analytic estimation of $\gamma$ since the coefficient $\left(q k_{r} \rho_{t i}\right)^{2}$ in Eq. (26) is expected to be very small in the benchmark case. One can see the frequency and damping rate of the $E_{r}$ and $\Gamma_{i}$ oscillation agree well among two simulation results and the analytic estimation. Moreover, good agreements are also found in the amplitude of the GAM oscillation, time evolution of non-oscillation part of $E_{r}$, and the equilibrium value of it.

Finally, the radial profile of the GAM frequency is compared for the case 1. Figures 8 (a) and (b) show the power spectrum of $E_{r}$ oscillation in GT5D and FORTEC-3D, respectively. The power spectrum is taken between the range $0 \leq t / \tau_{i i}<0.32$ and normalized on each flux surface. In both figures, the analytic evaluation of $\omega_{G}$ of Eq. (25) is also shown. It can be seen that a peak of the power spectrum appears at the same frequency as the theory predicts. The peak disappears in both simulations at the inside of the plasma, because strong Landau damping of GAM occurs at that region where the safety factor $q$ is close to unity. These results concerning the GAM oscillation and damping prove that both simulation codes can correctly solve not only the steady state neoclassical transport but also the transient phenomena that are inherent in the drift-kinetic equation.

\section{Conclusion}

In this work, detailed benchmark tests of neoclassical transport simulation in a tokamak plasma have been carried out for GT5D gyrokinetic code by comparing calculation results with those from FORTEC-3D drift-kinetic code and analytic estimations from neoclassical transport theory. On the whole, good agreements between the two simulations are found in the neoclassical heat diffusivity, parallel force balance relation, and GAM frequency and damping rate in a wide range of effective collisionality $0.01 \leq v_{*} \leq 10$. The radial profile of transport coefficients such as $\chi_{i}, k$-factor, 
and GAM frequency also coincide between two simulations. The simulation results are shown to agree with the theoretic values of them, while we have also found the finite-aspect-ratio and finite-orbit-width effects in some cases, which are neglected or approximated in analytic formulae but correctly taken into account in these two simulations.

The benchmark results shown here prove that GT5D code properly treats the neoclassical transport phenomena in its gyrokinetic full- $f$ Vlasov calculation, not only qualitatively, but also quantitatively. The conservation property of the linear Fokker-Planck collision operator adopted in the code, which is essentially the same one as FORTEC-3D uses, ensures capability of long-duration simulation required to see the steady state turbulence in a system with source and sink terms. And the adaptability to a wide range of collisionality parameter $v_{*}$ shown here is also important for the full-volume global transport code, since $v_{*}$ may vary largely from the plasma core to the edge.

On the other hand, the benchmark results also shows that FORTEC-3D code can solve the time evolution the radial electric field and the plasma distribution function according to the drift-kinetic equation, which change in as fast time scale as the GAM oscillation. This guarantees the usage of the code to investigate the dependence of GAM frequency and damping rate on magnetic field configuration as reported in Ref. [28]. Since it is usually time consuming to conduct a global gyrokinetic simulation, especially in non-axisymmetric configurations, it is beneficial to replace a gyrokinetic code with more simple FORTEC-3D code in researching the properties of GAM oscillation in realistic magnetic field configurations.

\section{Acknowledgments}

One of the authors (S.S.) would like to thank Dr. Ryutaro Kanno for useful comments on neoclassical transport simulation. He was supported by The Masaji Yoshikawa Prize for Fusion Energy 2008 from Japan Atomic Energy Agency (JAEA). This work was performed with the support and under the auspices of the NIFS Collaborative Research Program NIFS08KDAD008, NIFS09KTAD006 and NIFS08KNXN119. All the computation of GT5D was performed on the JAEA Altix3700Bx2 system.

\section{References}

[1] F. L. Hinton and R. D. Hazeltine, Rev. Mod. Phys. 48 (1976) 239.

[2] S. P. Hirshman and D. J. Sigmar, Nuclear Fusion 21 (1981) 1079.

[3] P. Helander and D. J. Sigmar, Collisional Transport in Magnetized Plasmas, Cambridge: Cambridge University Press (2002)

[4] A. J. Brizard and T. S. Hahm, Rev. Mod. Phys. 79 (2007) 421.

[5] H. Sugama, Phys. Plasmas 7 (2000) 466.

[6] D. E. Hastings, W. A. Houlberg, and K. C. Shaing, Nuclear Fusion 25 (1985) 445

[7] M. Wakatani, Stellarators and Heliotron Devices, Oxford Univ. Press, New York (1998) Chap. 7.

[8] Y. Idomura, H. Urano, N. Aiba, and S. Tokuda, Nuclear Fusion 49 (2009) 065029.

[9] X. Q. Xu and M. N. Rosenbluth, Phys. Fluids B 3 (1991) 627.

[10] Z. Lin, W. M. Tang, and W. W. Lee, Phys. Plasmas 2 (1995) 2975.

[11] M. N. Rosenbluth, P. H. Rutherford, J. B. Taylor, E. A. Frieman, and L. M. Kovrizhnikh, Plasma Physics and Controlled Nuclear Fusion Research Vol.1 (1971) (IAEA-CN-28/C-12)

[12] C. S. Chang and F. L. Hinton, Phys. Fluids 25 (1982) 1493.

[13] S. Satake, M. Okamoto, N. Nakajima, H. Sugama, M. Yokoyama, and C. D. Beidler, Nuclear Fusion 45 (2005) 1362.

[14] S. Satake, R. Kanno, and H. Sugama, Plasma and Fusion Research 3 (2008) S1062.

[15] Y. Idomura, M. Ida, S. Tokuda, and L. Villard, J. Comput. Phys. 226 (2007) 244.

[16] X. Zhong, J. Comput. Phys. 128 (1996) 19.

[17] A. H. Boozer, Phys. Fluids 24 (1981) 1999.

[18] R. G. Littlejohn, J. Plasma Phys. 29 (1983) 111

[19] R. B. White, Phys. Fluids B 2 (1990) 845.

[20] W. X. Wang et al., Plasma Phys. Control. Fusion 41(1999) 1091.

[21] S. Brunner et al., Phys. Plasmas 6 (1999) 4504.

[22] C. D. Beidler et al., Plasma Phys. Control. Fusion 43 (2001) 1131.

[23] M. Yu. Isaev, S. Brunner, W. A. Cooper, T. M. Tran, A. Bergamnn, C. D. Beidler, J. Geiger, H. Massgerg, J. Nührenberg, and M. Schmidt, Fus. Sci. Technol. 50 (2006) 440.

[24] J. P. Freidberg, Ideal Magneto-Hydro-Dynamics, Plenum Press, New York (1987) Chap. 6.

[25] S. P. Hirshman, Phys. Fluids 21 (1978) 1295

[26] H. Sugama and T. -H. Watanabe, J. Plasma Physics 72 (2006), 825.

[27] S. Satake, M. Okamoto and H. Sugama, Phys. Plasmas 9 (2002) 3946.

[28] S. Satake, H. Sugama, and T. -H. Watanabe, Nuclear Fusion 47 (2007) 1258. 


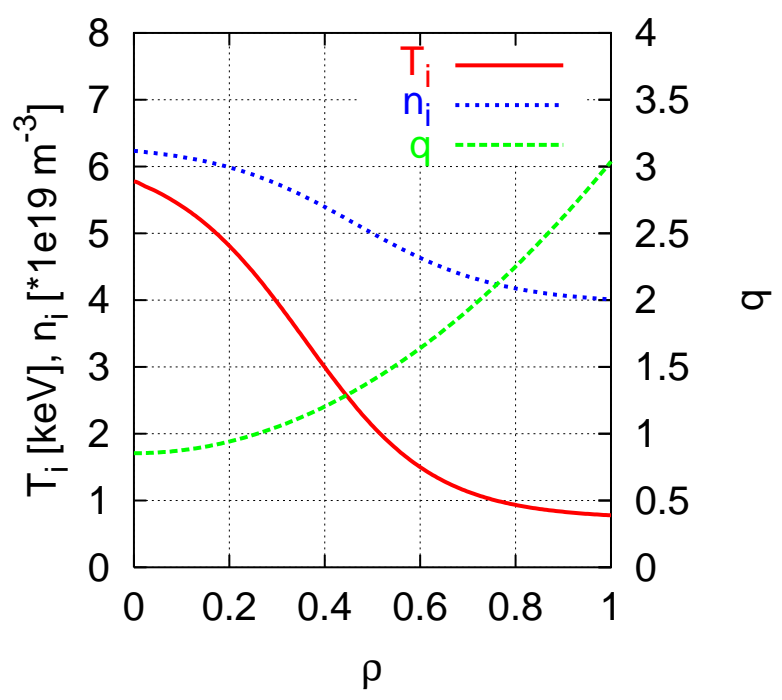

Figure 1: Ion density, temperature, and the safety-factor $(q)$ profiles in case 1.

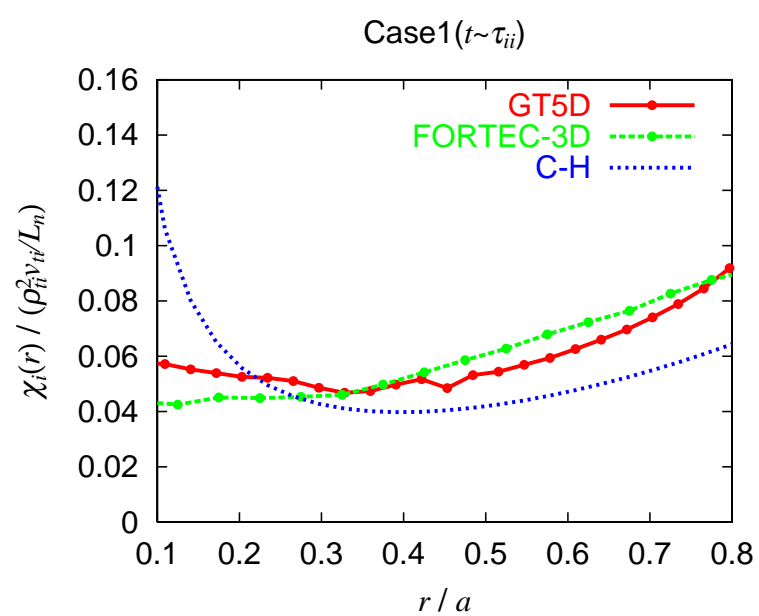

Figure 2: The radial profile of the ion heat diffusivity $\chi_{i}$ in the case 1. A dashed blue curve shows the estimation by the Chang-Hinton's formula.

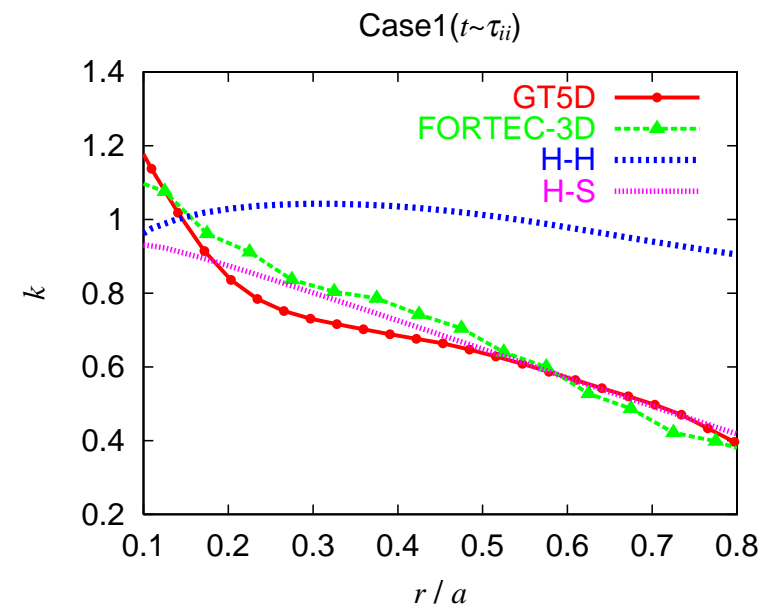

Figure 3: The radial profile of the force balance factor $k$ evaluated from $\left\langle U_{\| i} B\right\rangle$ and $E_{r}$ in the case 1. A dashed blue curve shows $k$ estimated by Hinton-Hazeltine's small-aspect-ratio formula (21), and a dotted purple line is obtained from Hirshman-Sigmar's moment equation approach in Ref. [2], respectively. 
Case2,3 $\left(t \sim \tau_{i i}\right)$, Case4 $\left(t \sim 2 \tau_{i i}\right)$, Case5 $\left(t \sim 8 \tau_{i i}\right)$

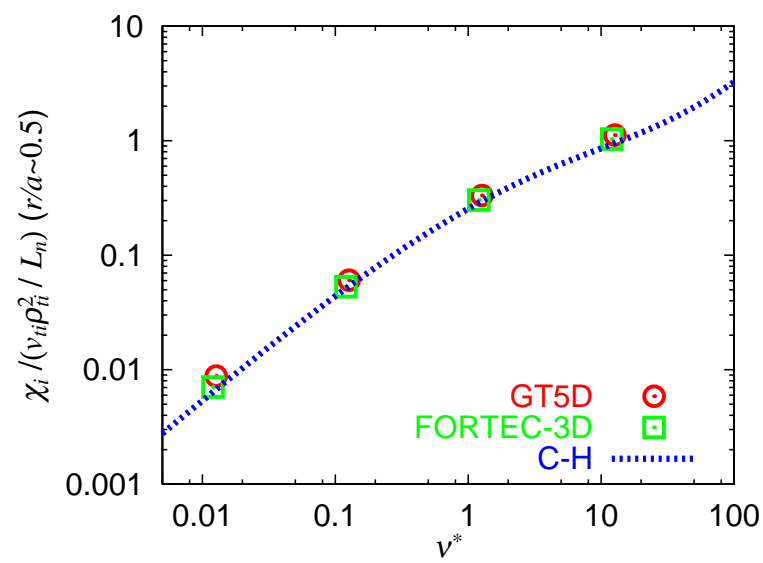

Figure 4: The collisionality dependence of the ion heat diffusivity $\chi_{i}$ in the cases 2 to 5 at $r=0.5 a$. A dashed blue curve shows the estimation by the Chang-Hinton's formula.

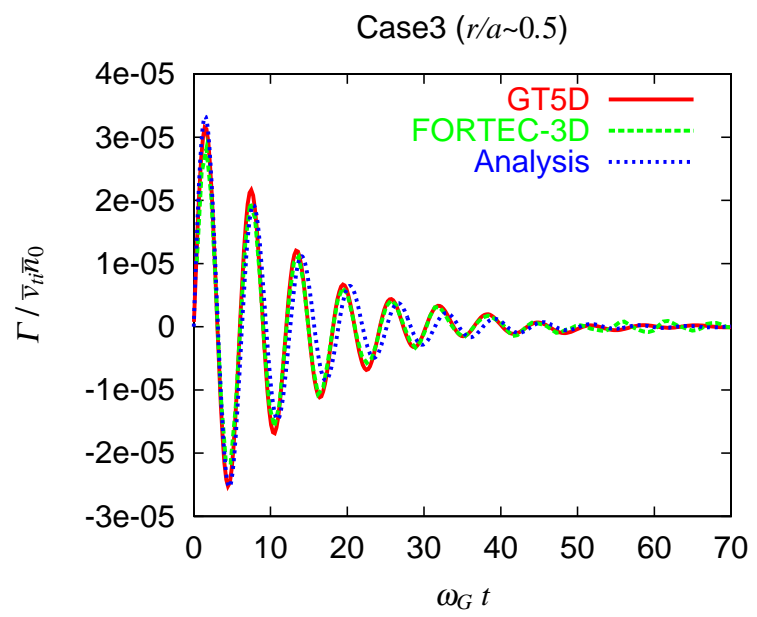

Figure 6: The comparison of radial ion particle flux $\Gamma_{i}$ observed in GT5D and FORTEC-3D codes at $r=0.5 a$ flux surface in the case 3. The horizontal axis is a time normalized by the GAM frequency $\omega_{G}$, which is evaluated by analytic formula Eq. (25). The vertical axis is normalized by volume-averaged density and thermal velocity. An analytic estimation of GAM oscillation $\Gamma(t)=\Gamma_{0} \sin \left(\omega_{G} t\right) \exp (\gamma t)$ is also plotted here with dotted curve, where the damping rate $\gamma$ is estimated by Eq. (26). In each simulation, GAM oscillation damps toward the ambipolar condition $\Gamma_{i}=0$ with almost the same damping rate and frequency with the analytic estimations.
Case2,3 $\left(t \sim \tau_{i i}\right)$, Case4 $\left(t \sim 2 \tau_{i i}\right)$, Case5 $\left(t \sim 8 \tau_{i i}\right)$

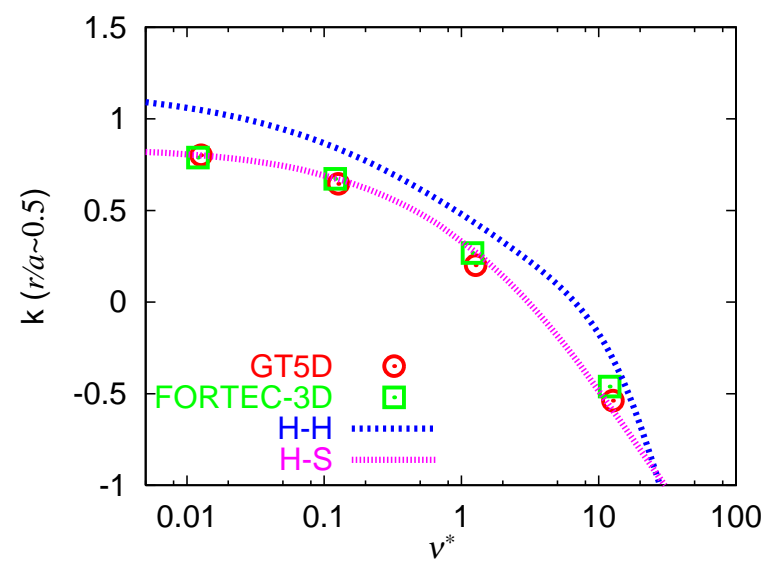

Figure 5: The collisionality dependence of the force balance factor $k$ evaluated from $\left\langle U_{\| i} B\right\rangle$ and $E_{r}$ in the cases 2 to 5 at $r=0.5 a$. A dashed blue curve shows $k$ estimated by Hinton-Hazeltine's formula (21), and a dotted purple line is obtained from Hirshman-Sigmar's moment equation approach in Ref. [2], respectively.

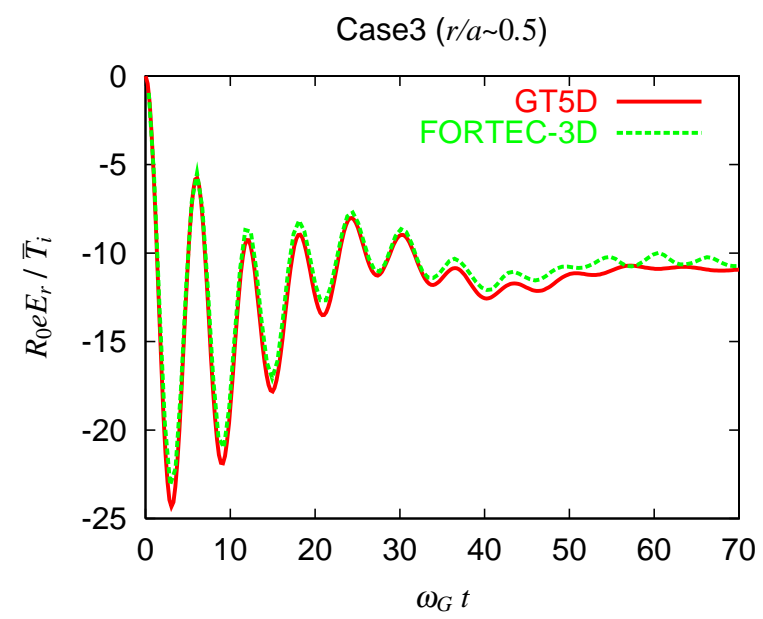

Figure 7: The comparison of radial electric field $E_{r}$ observed in GT5D and FORTEC-3D codes at $r=0.5 a$ flux surface in the case 3 . The horizontal axis is normalized as in Fig. 6, and the vertical axis is normalized by $R_{0} e / \bar{T}_{i}$ where $\bar{T}_{i}$ is the volume-averaged value. After transient damping phase, $E_{r}$ reaches a equilibrium state where the non-zero poloidal $\mathbf{E} \times \mathbf{B}$ flow is developed self-consistently to satisfy the neoclassical force balance relation. 
(a) : GT5D, $E_{r}$

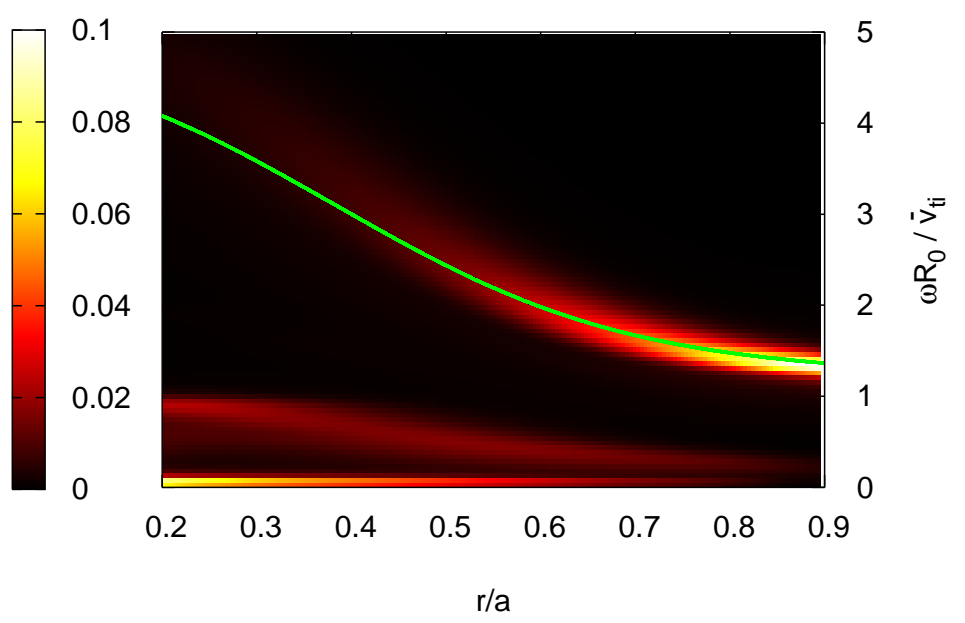

(b) : FORTEC-3D, $E_{\mathrm{r}}$

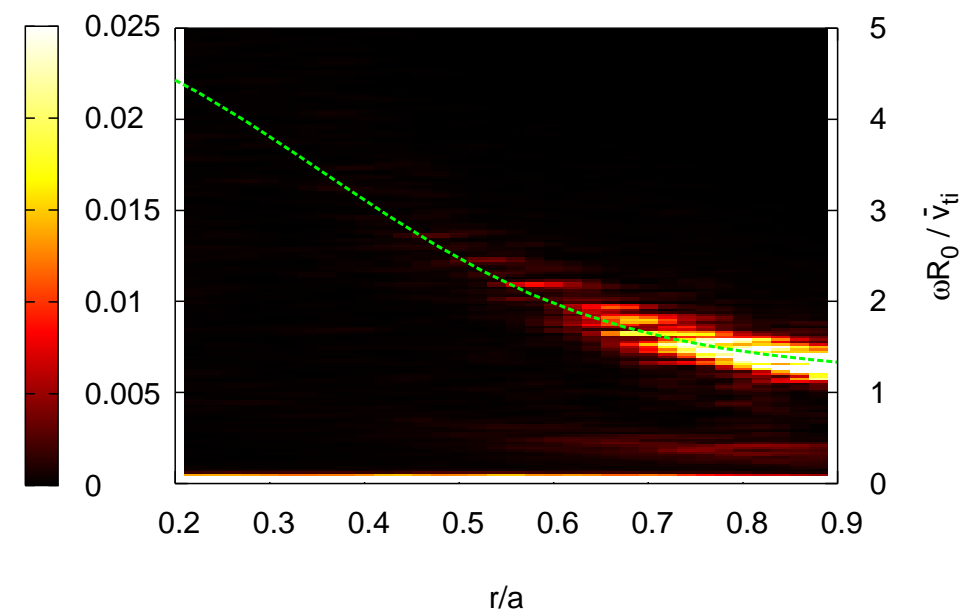

Figure 8: The power spectra of $E_{r}$ oscillation for the case 1 in (a): GT5D and (b): FORTEC-3D, respectively. The frequency is normalized by $R_{0} / \bar{v}_{t i}$, where $\bar{v}_{t i}$ is a volume-averaged thermal velocity. The color contour shows the intensity of the spectrum, which is normalized on each radial position, and a solid curve represents the analytic estimation for a tokamak, Eq. (25). 